Fifth Day: Fri., Nov. 11

Morning Session General Circulation I

Some Critical Parameters which Determine the Form on the General Circulation of the Atmospheres of the Earth and Other Planets

Y. Mintz

\title{
Some Results from a Theoretical Model of Vertical Heat Transfer
}

\section{S. Manabe and F. Möller}

The results of the numerical computation of thermal equilibrium temperature will be presented. The thermal processes taken into consideration are the absorption of solar radiation by water vapor, cloud, ozone and carbon dioxide, the transfer of long wave radiation due to these gases, the heat conduction from the earth's surface to the atmosphere, the condensation process.

The thermal equilibrium solution was obtained as the asymptotic solution of initial value problem.

These computations give us a good estimate of the sensitivity of the thermal equilibrium temperature to various factors. The computed latitudinal distribution of thermal equilibrium temperature reveals some of the major features of actual temperature distribution of the stratosphere as well as of the troposphere and their seasonal variation.

\section{A Numerical Experiment Using Five-Level Geostrophic Model}

\section{S. Matsumoto}

An integration is made by using a five-level geostrophic model with zonally cyclic and meridionally symmetric boundary condition. The functional form of heating is assumed to be proportional to $\mathrm{T}^{*}-\mathrm{T}$, where $\mathrm{T}^{*}$ is an equilibrium temperature.

A realistic general flow is generated after 40 days starting from a calm atmosphere. Having thus obtained the general flow, a random disturbance is applied on the middlelevel geopotential field and integration is continued for 12 days thereafter.

Three-cell circulation in the meridional plane develops for several days, and then it turns into five cells. Frontal layers and a well organized disturbance with a wave length of $4000-6000 \mathrm{~km}$ are observed to develop, and the latter shows a remarkable occlusion process.

Some remarks will also be given concerning another integration made under the influence of zonally differential heating. 